\title{
Lessons of the month 3: Duodenal perforation after polystyrene sulfonate
}

\author{
Authors: Winston WS Fung, ${ }^{\mathrm{A}}$ Wai Kin Chi, ${ }^{\mathrm{B}}$ Cheuk Chun Szeto, ${ }^{\mathrm{C}}$ Philip K-T Li ${ }^{\mathrm{D}}$ and Kai Ming Chow ${ }^{\mathrm{E}}$
}

Ion-exchange resins, sodium or calcium polystyrene sulfonate, are commonly used medications for management of hyperkalaemia. However, the drug can be associated with serious bowel injury. We report a case of a renal transplant recipient who developed duodenal ulcer perforation secondary to the use of calcium polystyrene sulfonate. Characteristic eosinophilic non-polarisable rhomboid shaped crystals were evident in the affected area of ulceration on histologic examination in addition to features of cytomegalovirus inclusions. We also hypothesised that gastroparesis secondary to autonomic dysfunction could have led to prolonged luminal contact time with polystyrene, further predisposing to bowel injury.

KEYWORDS: Hyperkalaemia, intestinal perforation, renal transplant, cytomegalovirus, polystyrene

\section{Case presentation}

A 45-year-old man presented with sudden onset of abdominal pain and passing tarry stool. He was diagnosed to have diabetes mellitus at the age of 30 years followed by a myriad of autonomic complications including postural hypotension and gastroparesis. His past medical history also included hypertension, hyperlipidaemia and end-stage renal failure treated with pre-emptive cadaveric renal transplant 3 months prior to index admission. Immunosuppression included prednisolone, tacrolimus and mycophenolate mofetil. The kidney transplant was complicated with delayed graft function requiring temporary dialysis. After renal biopsy diagnosis of antibody-mediated rejection, he was treated with plasma exchange to stabilise the graft function, with serum creatinine around $300 \mu \mathrm{mol} / \mathrm{L}$. He had recurrent hyperkalaemia requiring treatment.

During the acute presentation with passing tarry stool, laboratory studies revealed a potassium level of $6.0 \mathrm{mmol} / \mathrm{L}$ and haemoglobin of $4.7 \mathrm{~g} / \mathrm{dL}$. The patient denied use of non-steroidal

Authors: ${ }^{\text {A }}$ resident specialist, Prince of Wales Hospital, Hong Kong; ${ }^{B}$ resident specialist, Prince of Wales Hospital, Hong Kong; ${ }^{C}$ lecturer, The Chinese University of Hong Kong, Hong Kong; ${ }^{D}$ consultant, Prince of Wales Hospital, Hong Kong; ${ }^{E}$ consultant, Prince of Wales Hospital, Hong Kong anti-inflammatory drugs. Oesophagealgastroduodenoscopy then showed a $2 \mathrm{~cm}$ ulcer at the first part of duodenum with eroded edge and necrotic base (Fig 1). A biopsy of the ulcer was performed. He further developed severe and persistent abdomina pain; laparotomy exploration showed a perforated duodenal ulcer requiring omental patch repair. Histology of the duodenal ulcer biopsy specimen (Fig 2), in retrospect, revealed nonpolarisable rhomboid shaped crystals (yellow triangle) among necrotic slough (yellow asterisk) while cytomegalovirus (CMV) inclusions (yellow arrow) were evident in the endothelial cells and stromal cells. There was no evidence of recent vascular thrombosis or fibrin or cholesterol emboli. On further questioning, the patient had received repeated doses of calcium polystyrene sulfonate for hyperkalaemia 1 week prior to the onset of symptoms. The patient recovered from the surgery and was discharged after completing a course of ganciclovir treatment; there was no further use of calcium polystyrene sulfonate.

\section{Discussion}

Gastrointestinal perforation affects the colon more often than duodenum following kidney transplantation. ${ }^{1}$ The duodenal ulcer

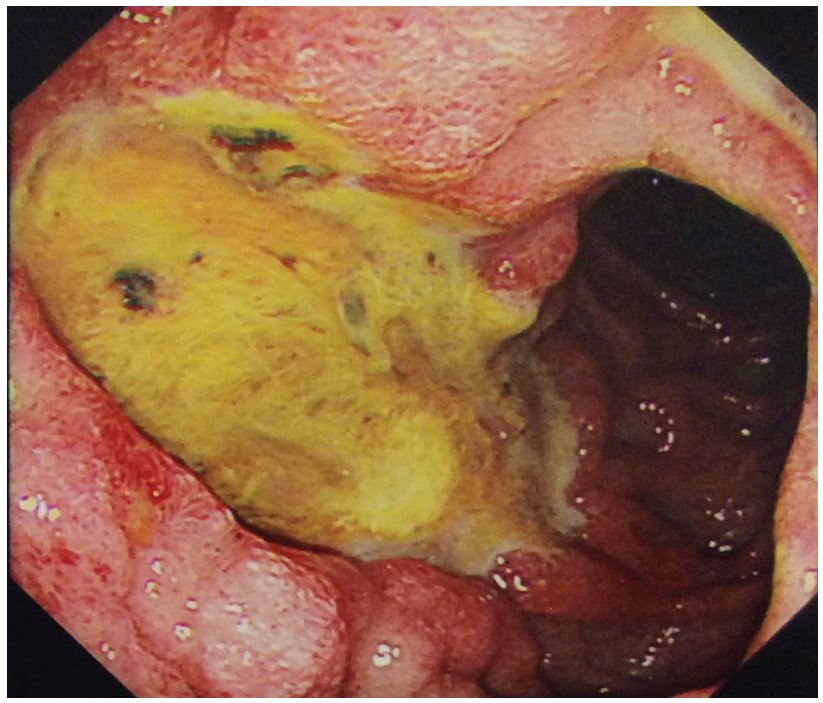

Fig 1. Endoscopy showed a $2 \mathrm{~cm}$ ulcer at first part of duodenum with eroded edge and necrotic base. 


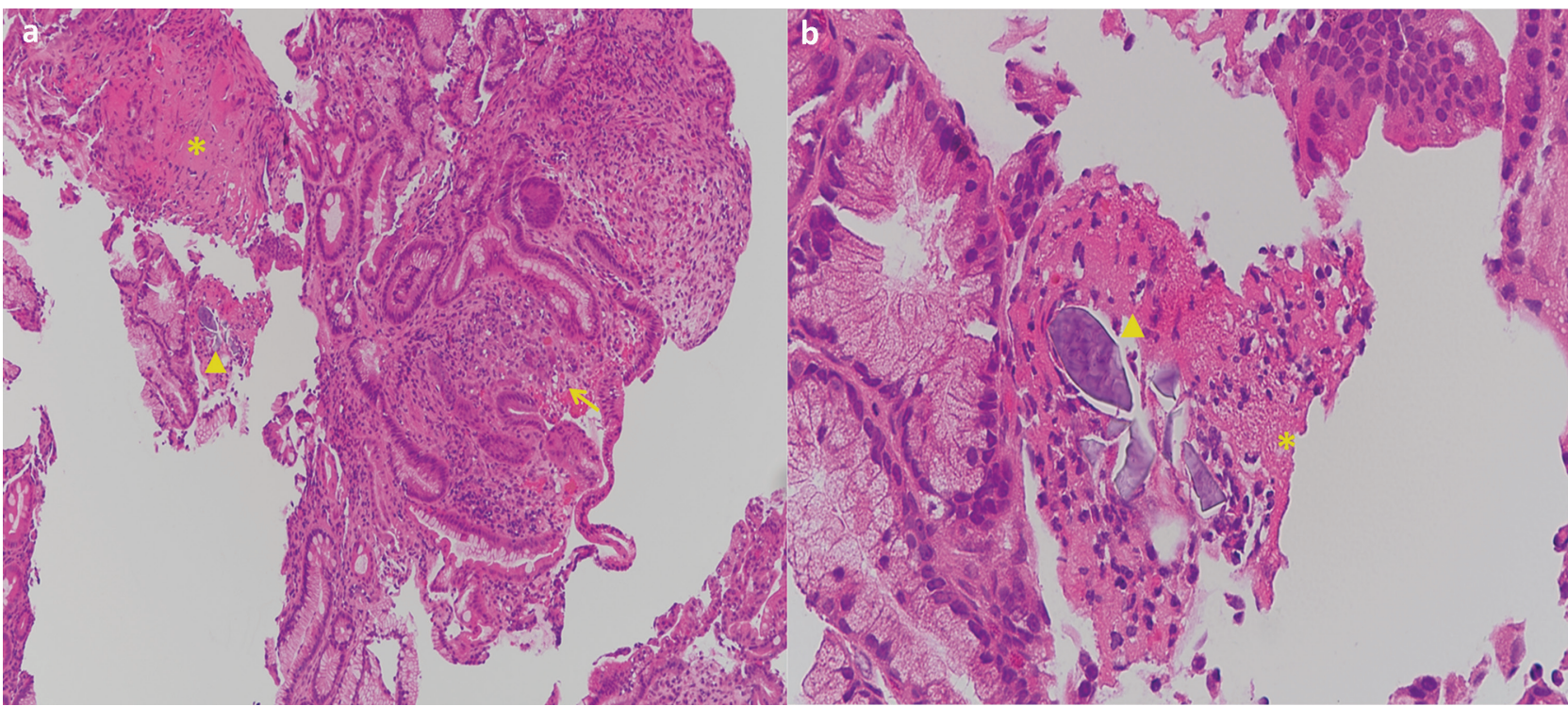

Fig 2. Biopsy specimen of the duodenal ulcer stained with haematoxylin and eosin. Nonpolarisable rhomboid shaped crystals (yellow triangle) among necrotic slough (yellow asterisk) with cytomegalovirus inclusions in endothelial cells and stromal cells (yellow arrow) a) at 200x magnification and b) at $400 x$ magnification.

causing perforation in this patient can be multifactorial. Although ulcerogenic effect of corticosteroid is controversial, high dose of steroid use during early transplant and treatment of rejection in this patient could have contributed to haemorrhagic or stress ulcers in this patient. Further background information should have been elicited from this patient, including history of Helicobacter pylori infection, pre-existing gastroduodenal ulcer and healing status. Other transplant-specific conditions that are more common at the early period of kidney transplantation leading to duodenal perforation include CMV infection and post-transplant lymphoproliferative disorder involving the gastrointestinal system.

This patient was at high risk of CMV infection given the early post-transplant status and rejection treatment. The most common clinical manifestation of tissue-invasive CMV disease in kidney transplant recipients is gastrointestinal disease, affecting at least half of the cases. ${ }^{2}$ These CMV related gastrointestinal manifestation include diffuse mucosal inflammation leading to gastritis/colitis, haemorrhage, ulcer or even perforation, as shown by this case. Diagnosis is usually suggested by quantitative viral load based on nucleic acid testing using the CMV polymerase chain reaction, although a biopsy with histopathologic examination of tissue is more confirmatory. Endoscopically, the lesions could range from patchy erythema or exudates to deep ulcers (Fig 1). Characteristic histopathological findings are cytoplasmic inclusions and nuclear enlargement with eosinophilic nuclear inclusions.

The most striking abnormality of the biopsy, in fact, showed abundant calcium polystyrene sulfonate crystals among necrotic slough. Differential diagnosis of calcium polystyrene sulfonate or sodium polystyrene sulfonate crystals includes cholestyramine, a bile acid sequestrant. Cholestyramine, however, was not used by our patient and its crystals are rhomboid shaped and stained bright orange-red on haematoxylin and eosin stain. Furthermore, they do not cause mucosal injury, in contrast to polystyrene sulfonate crystals (which are commonly found aggregated within the injured areas of the gastrointestinal tract specimens). In the context of current clinical and histopathological features, it is suggestive of intestinal necrosis secondary to the toxic effect of cation exchange resin polystyrene sulfonate.

Sodium polystyrene sulfonate, or calcium polystyrene sulfonate depending on drug availability in different countries, is a common treatment option of hyperkalaemia. In addition to its poor gastrointestinal side effect profile, polystyrene sulfonate can cause serious gastrointestinal complications. Polystyrene sulfonate-associated small-bowel and colon ulceration or even necrosis can occur, with and without concomitant sorbitol. ${ }^{3}$ By 2005, 47 years after sodium polystyrene sulfonate approval by the US Food and Drug Administration, there were 35 adverse event reports of serious bowel injuries in association with either oral administration or enema of this medication, many of them fatal. ${ }^{4}$ A large population-based matched cohort study recently showed that sodium polystyrene sulfonate use was associated with a nearly two-fold increased risk of hospitalisation within 30 days of initial prescription for adverse gastrointestinal events, including intestinal ischaemia or thrombosis, ulceration or perforation, requiring bowel resection. ${ }^{5}$ Reported pathological features of the condition range from patchy mucosal erosions or ulcerations to pseudomembranes to transmural necrosis. ${ }^{6}$

Based on previous literature and systematic review, risk factors of this condition include acute kidney injury, chronic kidney disease, solid organ transplantation and postoperative stress. ${ }^{3}$ High renin levels have been postulated to be the causative mechanism, whereby patients develop nonocclusive mesenteric ischaemia via angiotensin-mediated vasoconstriction. The concurrent gastroparesis and delayed gastrointestinal transit secondary to his autonomic dysfunction, supported by the repeated endoscopic finding of retained food residues in this patient (Fig 1), could have led to prolonged luminal contact time with polystyrene. 6 Given the background of underlying CMV colitis and immunosuppressed state, administration of calcium polystyrene sulfonate could have triggered the gastric injury and eventually perforation. ${ }^{7}$. 


\section{Key points}

> Accumulating evidence suggests that patients receiving traditional potassium binding resin, polystyrene sulfonate, are at risk for serious gastrointestinal adverse events such as intestinal necrosis.

> Benefits of hyperkalaemia treatment with polystyrene sulfonate should be balanced against concerns about serious bowel injury associated with the drug.

\section{References}

1 Catena F, Ansaloni L, Gazzotti F et al. Gastrointestinal perforations following kidney transplantation. Transplant Proc 2007;40:1895-6.

2 Helanterä I, Schachtner T, Hinrichs C et al. Current characteristics and outcome of cytomegalovirus infections after kidney transplantation. Transpl Infect Dis 2014;16:568-77.

3 Harel Z, Harel S, Shah PS et al. Gastrointestinal adverse events with sodium polystyrene sulfonate (Kayexalate) use: a systematic review. Am J Med 2013;126:264.e9-24.
4 Sterns RH, Rojas M, Bernstein P, Chennupati S. Ion-exchange resins for the treatment of hyperkalemia: are they safe and effective? J Am Soc Neprhol 2010;21:733-5.

5 Noel JA, Bota SE, Petrcich W et al. Risk of hospitalization for serious adverse gastrointestinal events associated with sodium polystyrene sulfonate use in patients of advanced age. JAMA Intern Med 2019;179:1025-33.

6 Rashid A, Hamilton SR. Necrosis of the gastrointestinal tract in uremic patients as a result of sodium polystyrene sulfonate (Kayexalate) in sorbitol: an underrecognized condition. Am J Surg Pathol 1997:21:60-9.

7 Gorospe EC, Lewis JT, Bruining DH. Kayexalate-induced esophageal ulcer in a patient with gastroparesis. Clin Gastroenterol Hepatol 2012:10:A28.

8 Gürtler N, Hirt-Minkowski P, Brunner SS et al. Sodium polystyrene sulfonate and cytomegalovirus-associated duodenitis: more than meets the eye. Am J Case Rep 2018;19:912-6.

Address for correspondence: Dr Kai Ming Chow, Department of Medicine and Therapeutics, Prince of Wales Hospital, The Chinese University of Hong Kong, Shatin, Hong Kong.

Email: chow_kai_ming@alumni.cuhk.net

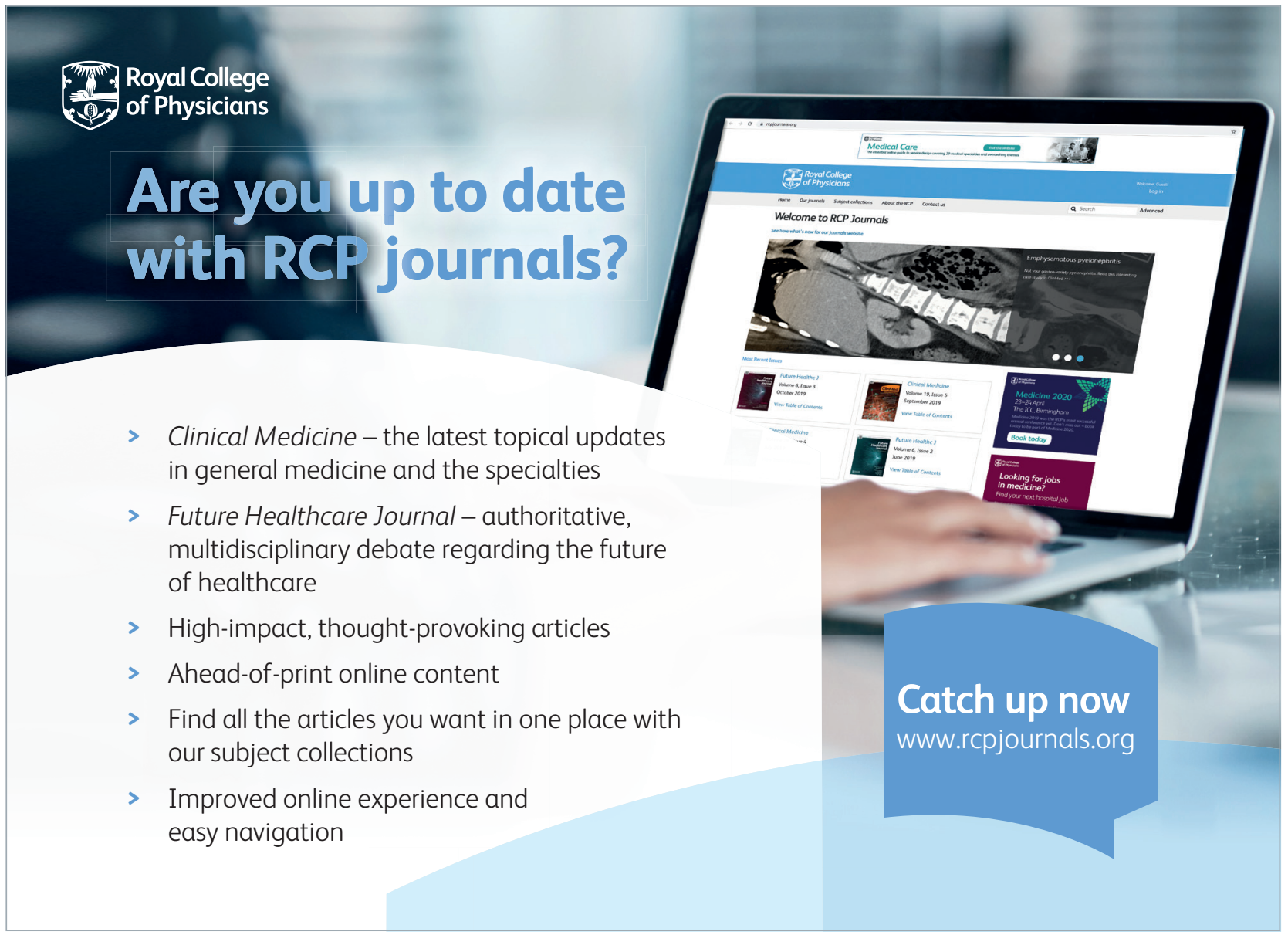

